\title{
Performance Comparison between Delay-Tolerant and Non-Delay-Tolerant Position-Based Routing Protocols in VANETs
}

\author{
Ahmed Mohamed Abdalla, Salem H. Salamah \\ College of Technological Studies, PAAET, Kuwait City, Kuwait \\ Email: am.mahmoud@paaet.edu.kw, sh.salamah@paaet.edu.kw
}

\begin{abstract}
How to cite this paper: Abdalla, A.M. and Salamah, S.H. (2022) Performance Comparison between Delay-Tolerant and Non-DelayTolerant Position-Based Routing Protocols in VANETs. Int. J. Communications, Network and System Sciences, 15, 1-14.

https://doi.org/10.4236/ijcns.2022.151001
\end{abstract}

Received: December 16, 2021

Accepted: January 15, 2022

Published: January 18, 2022

Copyright $\odot 2022$ by author(s) and Scientific Research Publishing Inc. This work is licensed under the Creative Commons Attribution International License (CC BY 4.0).

http://creativecommons.org/licenses/by/4.0/

\begin{abstract}
Vehicular ad hoc networks (VANETs) are a new emerging recently developed advanced technology that allows a wide group of applications related to providing more safety on roads, more convenience for passengers, self-driven vehicles, and intelligent transportation systems (ITS). There are various routing protocol categories used in VANETs, like unicast, multicast, and broadcast protocols. In unicast position-based protocols, the routing decisions are based on the geographic position of the vehicles. This does not require establishment or maintenance of routes but needs location services to determine the position of the destination. Non-delay-tolerant network protocols (non-DTN), also identified as minimum delay protocols, are aimed at minimizing the delivery time of the information. Delay-tolerant protocols (DTN) are used in a variety of operating environments, including those that are subject to failures and interruptions and those with high delay, such as VANETs. This paper discusses the comparison between non-DTN and DTN routing protocols belonging to the unicast delay-tolerant position-based category. The comparison was conducted using the NS2 simulator, and the simulations of three non-DTN routing protocols and three DTN routing protocols were recorded. Simulation results show that the DTN routing protocols outperform in delivery ratio compared to the non-DTN routing protocols, but they lead to more average delay due to buffering, the processing algorithm, and priority calculation. In conclusion, non-DTN protocols are more suitable for the city environment since the distance between nodes is relatively smaller and the variations in the network topology are slower than they are on highways. On the other hand, DTN protocols are more suitable for highways due to the buffering of packets until a clear route to destination is available.
\end{abstract}




\section{Keywords}

VANETs, Position-Based Routing Protocols, Non-DTN, DTN

\section{Introduction}

Vehicular ad hoc networks (VANETs) are derived from the mobile ad hoc networks (MANETs). VANETS are established especially for car-to-car communication $(\mathrm{C} 2 \mathrm{C})$ between moving vehicles and/or roadside units (RSUs). VANETs allow for a wide group of applications that pertain to providing more safety on roads, more convenience for passengers, self-driven vehicles, and intelligent transportation systems (ITS) [1] [2].

Geographic routing protocols are considered more suitable for highly dynamic environments, such as VANETs. These protocols are commonly categorized into DTN and non-DTN [3]. Vehicular delay-tolerant network (VDTN) protocols are specifically designed to handle network partitioning and disconnections mainly caused by frequent mobility and sparse topology [4]. Hence, the packet delivery ratio in VDTN is more important than delay as these networks are characterized by inadequate transmission opportunities and irregular connectivity.

As the direct connection with a node in VDTN may not be possible because of the restricted transmission range of roadside units (RSUs), vehicles may be considered an intermediate node to relay packets [5].

The automobile industry is currently motivated by the requirement of self-driven vehicles; thus, there is a dire need for the exchange of information vehicles to enhance the safety, security, and convenience of drivers and passengers alike [3] [4] [5]. So far, we have three types of communications in VANETs, namely vehicle-to-vehicle (V2V) communication (i.e., communication between the self-driven vehicles themselves), vehicle-to-roadside infrastructure (V2I), and roadside infrastructure-to-roadside infrastructure (I2I).

Routing in vehicular ad hoc networks involves many challenges in light of their specific features, such as the high mobility of vehicles, the topological dynamic changes, and the highly segregated network. These features are commonly regarded as challenging in our pursuit to achieve a reliable, non-stop, and seamless way to communicate in the presence of moving vehicles.

In unicast routing protocols, data is transmitted from one source to one destination. This is the basic unit protocol in ad hoc networks on which other types of protocols are based. Subdivisions are further made from unicast routing protocols according to topology, position, cluster, and hybrid protocols.

In position-based routing protocols, all vehicles can recognize their own locations and the geographic locations of their neighbor's vehicle through position-pointing devices, such as a global positioning system (GPS). A GPS device does not make any routing table or exchange any information related to 
the status of the link with the neighbor vehicles; rather, it provides the information used in making routing decisions. This type of routing provides better performance because there is no need to create and maintain an overall route path from the source vehicle to the destination vehicle. The position-based routing protocols may be further categorized into non-delay-tolerant network (non-DTN) routing protocols, delay-tolerant network (DTN) routing protocols, and hybrid routing protocols.

The paper is organized as follows. Section I introduces VANET and different routing protocols. Vehicular ad hoc networks are illustrated in Section II. Section III discusses the issues related to routing protocols in VANETs. Section IV introduces VANETs unicast position-based routing protocols. Sections V and VI discuss DTN and non-DTN protocols. Section VII includes the simulation results and discussion. Finally, Section VIII presents the conclusions of the study.

\section{Vehicular AD HOC Networks}

Vehicular ad hoc networks (VANETs) can be classified as a subcategory of mobile ad hoc networks (MANETs) that act between the moving vehicles on one hand and the nearest fixed roadside units (RSUs) on the other. VANETs are a high-end emerging technology, extensively used as means to provide more safety on roads and more convenience for passengers; it also serves self-driven vehicles and intelligent transportation systems (ITS) [1] [3].

Efficient routing protocols help make roads safer by rapidly disseminating information about the road conditions and traffic among the participating vehicles within a very short period. VANETs also enable automation of both city and highway applications so that vehicles can travel without receiving any assistance from drivers. These applications were once fantasies yet have become realistic, and the demand for them has risen. VANETs has some specific unique challenging features, such as high mobility of vehicles, varying density of vehicles based on time, highly segregated networks, frequent disconnections, and topological dynamic changes [6]. It is a challenge to build strong networks between vehicles and ensure continuous, secure, and reliable communication paths among the neighbor vehicles in motion.

The Federal Communications Commission (FCC) has assigned a spectrum 75 $\mathrm{MHz}$ within $5.9 \mathrm{GHz}$ band for vehicle-to-vehicle (V2V) and vehicle-to-roadside (V2I) communication, known as dedicated short-range communications (DSRC). In addition, the Institute of Electrical and Electronics Engineers (IEEE) is working on a standard for wireless access in vehicular environments (WAVE), known as the IEEE 1609 family, which implies an architecture and a complementary, standardized set of services and interfaces that jointly allow security for both $\mathrm{V} 2 \mathrm{~V}$ and V2I wireless communications [6]. The basic components of VANETs are shown in Figure 1.

\section{Vanets Routing Protocols}

Achieving reliable and fast routing in VANETs is the challenge we face consi- 
dering the unique specific nature and features of the network, such as a dynamically changing topology, the high mobility of vehicles, and the highly partitioned network. In the presence of fast-moving vehicles, it is quite challenging to ensure reliable, continuous, and seamless communication [7]. Many external factors, such as road topology, and internal factors, such as vehicle mobility, control the performance of VANETs routing protocols, meaning that we are in dire need for a highly adaptive approach to deal with the dynamic circumstances. This can only be achieved by selecting the best routing strategies and using appropriate transmission and mobility models. Types of VANETs routing protocols are illustrated in Figure 2.

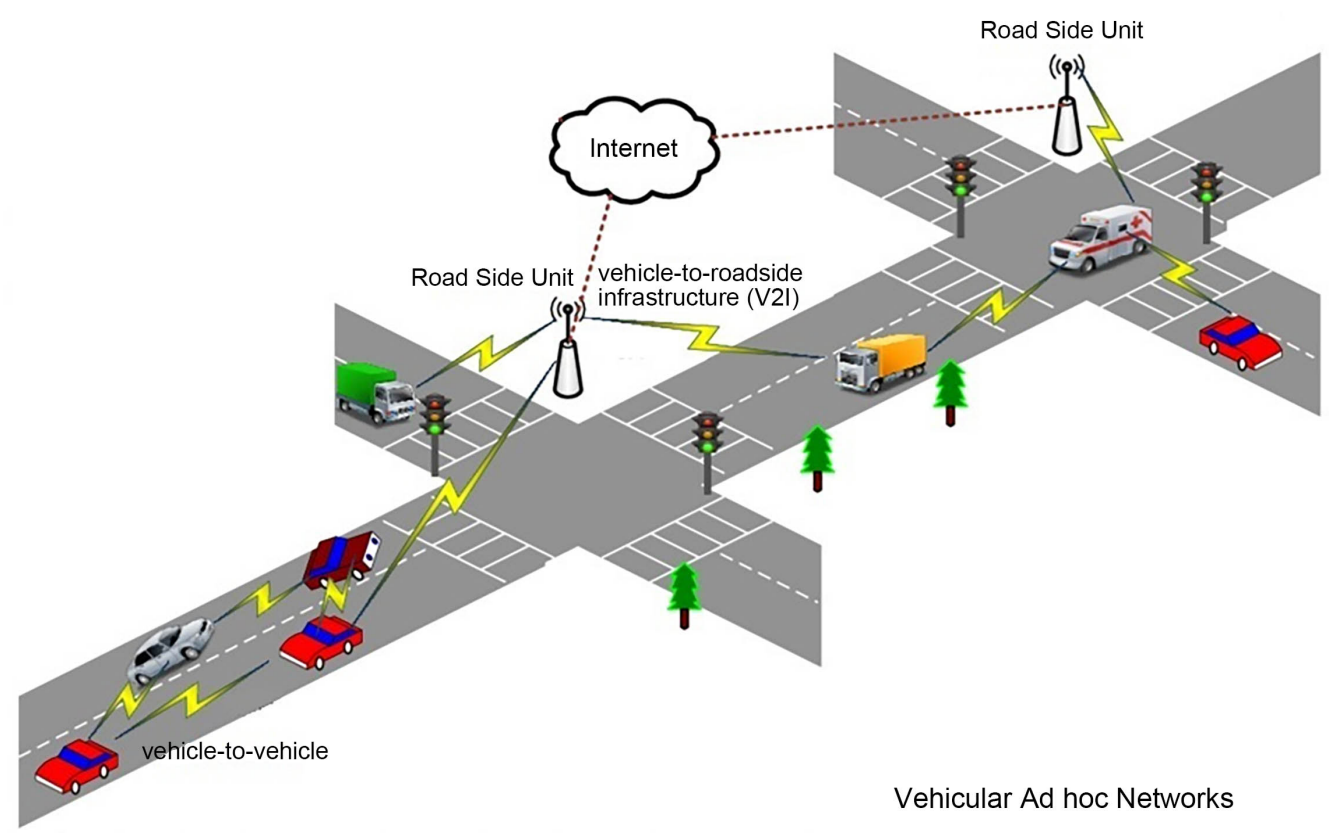

Figure 1. Vehicular ad hoc networks.

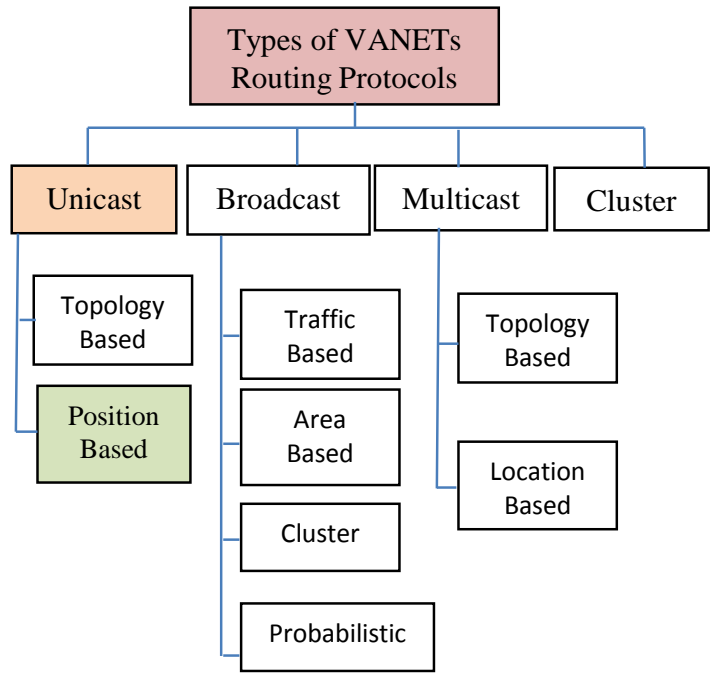

Figure 2. Types of VANETs routing protocols. 


\subsection{Unicast Routing Protocols}

Unicast routing protocols are commonly used to transmit data packets only from one vehicle source to one vehicle destination. These protocols are especially beneficial because they support both personalized and commercial applications, such as multimedia access and internet connectivity.

\subsection{Broadcast Routing Protocols}

Broadcast routing protocols are the routing protocols most used by VANETs, especially in safety-related applications. Among the remarkable techniques used with broadcast routing protocols is flooding; however, blind flooding creates a broadcast storm problem, meaning that the channel capacity is overloaded, leading to channel congestion and ultimately affecting the reliability of communication. Therefore, broadcast protocols are suitable only for a network with a limited number of vehicles [7] [8].

\subsection{Multicast/Geocast Routing Protocols}

Multicast routing allows for the transmission of messages from one source to a group of target destination vehicles. An example of location-based multicast routing is the geocast routing protocol, which aims to deliver information from only one source vehicle to all other vehicles inside a specified geographical region called a zone of relevance (ZOR) [9].

\subsection{Cluster Based Routing Protocols}

In vehicular ad hoc networks, clustering refers to the virtual partitioning of dynamic vehicles into several groups. Here, we have a group of vehicles known as clusters, and for each cluster, there is a cluster-head. Cluster-heads are assigned several responsibilities: channel assignment for cluster members, spreading inter-cluster traffic, scheduling intra-cluster traffic, and routing cluster members. In this protocol, the cluster members take no part in routing [10].

\section{Unicast Position-Based Routing Protocols}

The most prominent of all VANETs protocols are the unicast routing protocols in the ad hoc environment, on which other types of protocols are based. There are a number of subcategories that derive from the unicast routing protocols, based on topology, position, cluster, and hybrid protocols [11] [12].

Topology-based routing protocols proved to be unfeasible in VANETs due to the overheads related to the discovery of routes and the maintenance of routes in the presence of moving vehicles. Vehicle mobility is one of the most important factors affecting the VANET environment, as it leads to frequent network partitioning and route disconnection, which entail a recalculation of the propagation paths according to the new topology information.

In position-based protocols, the routing decisions are highly dependent on the geographic position of the vehicles, which demands no establishment or main- 
tenance of routes but rather requires location services to determine the position of the destination. Simple location services (SLS), reactive location services (RLS), DREAM location services (DLS), and global positioning systems (GPS) are some of the commonly used location services. In this protocol, data is transmitted regardless of the digital map to the one-hop neighbor, being the closest to the position of the destination vehicle. Therefore, beacon (Hello) packets with the vehicle position information and other vehicle identification parameters are frequently sent by each vehicle. Position-based protocols are suitable for VANETs and are better than topology-based routing protocols since they offer a higher delivery ratio in a highly mobile environment. Therefore, they have the advantage of providing minimum delay in establishing the route path and also achieve good scalability.

Greedy forwarding, contention-based forwarding, opportunistic forwarding, trajectory-based forwarding, and hybrid forwarding are examples of forwarding routing mechanisms used by position-based routing protocols.

In position-based routing protocols, with the use of through position-pointing devices, such as GPS, all vehicles know their own positions and their neighbor vehicle's geographic locations. A GPS device does not create a routing table and does not exchange connection status information with neighboring vehicles but instead provides the information that is used in routing decisions. This type of route provides better performance because it is not necessary to create and maintain a total route path from the source vehicle to the destination vehicle.

Position-based routing protocols may be further classified into non-delay-tolerant network (non-DTN) routing protocols, delay-tolerant network (DTN) routing protocols, and hybrid routing protocols [13] [14] [15] [16]. Figure 3 illustrates the classification of position-based routing protocols. In this paper, our focus is on the DTN routing protocols.

DTNs are used in a variety of operating environments, including those that are subject to outages and interruptions and those with high delay, such as vehicular ad hoc networks (VANETs).

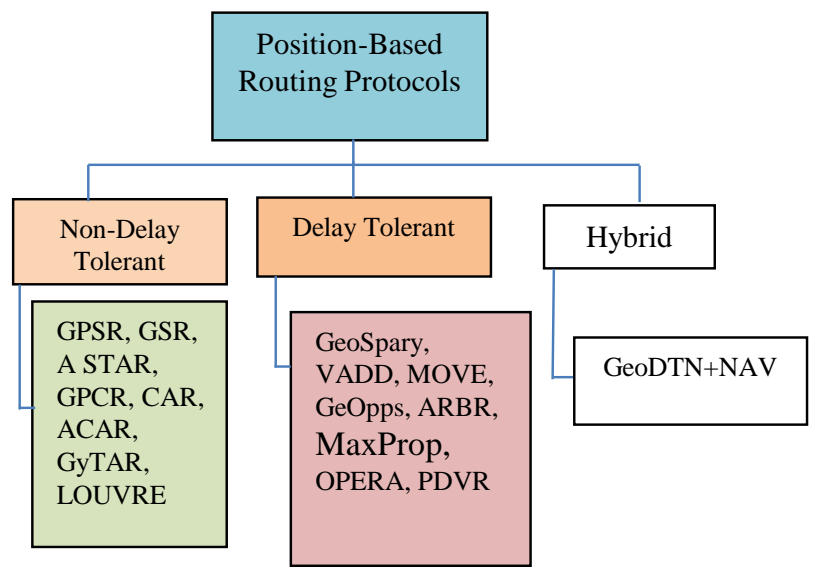

Figure 3. Classification of position-based routing protocols. 
Due to the limited transmission range of a RSU, remote vehicles may not be able to connect directly to the RSU and therefore must rely on intermediary vehicles to forward packets. During the message relay process, complete end-to-end paths may not exist in highly segmented VANETs. Therefore, the intermediate vehicles must use a buffer to store and forward messages opportunistically.

Through the buffer, carry, and forward, the message can finally be delivered to the destination, even if there is never an end-to-end connection between the source and the destination. The main objective of routing protocols in DTN is to maximize the probability of the delivery ratio to the destination while minimizing the end-to-end delay. In addition, vehicle traffic models are significant for DTN routing in vehicle networks because the performance of DTN routing protocols is closely related to the population and mobility models of the network.

In dynamic network environments, an adaptive framework and VDTN routing protocols are necessary to discover an appropriate next-hop forwarder node from neighbor on the path toward the destination. This selection should be made in a way that increases the data delivery probability with a reduction in the delay time and that balances the network overhead.

\section{Non-Delay-Tolerant Network (NON-DTN)}

Non-delay-tolerant network protocols are also identified as minimum delay protocols, and their goal is to minimize the delivery time of the information from source node to the destination node. Non-DTN protocols are suitable for use in real-time safety applications; they request a critical time response during data distribution. End-to-end delay time in packets transmission is the main parameter in designing non-DTN protocols, and the shortest path method is commonly implemented. However, the shortest path method may not always guarantee faster delivery, especially when the traffic conditions are light.

The greedy forwarding technique is commonly used in VANETs, in which the packet is forwarded to a neighbor node that is geographically closer to the destination node. The greatest problem occurs when a packet reaches a node that has no neighbors closer to the destination. A local maxima problem occurs, and each routing protocol in this group needs to apply its own recovery strategy to overcome the local maxima problem. One of the earliest protocols used in greedy protocols is greedy perimeter stateless routing (GPSR). GPSR makes greedy forwarding decisions only by using instant information about the neighbors of the routing vehicle in the network. When a local maxima problem occurs, GPSR uses the perimeter forwarding technique for recovery. GPSR uses many models for mobility prediction that consider the mobility of vehicle groups.

The GPSR routing protocol works well in highway environments but achieves low performance in city environments. GPSR relies on a distributed algorithm for graphs. For radio problems, such as tall buildings in urban areas, these algorithms often partition an otherwise connected graph, making the delivery of 
packets impossible.

Geographic source routing (GSR) merges position-based routing with topological knowledge. GSR gets the position information of the destination node using an RLS system and uses a city map for complete information of city topology. Greedy perimeter coordinator routing (GPCR) also takes advantage of the fact that city streets form a natural planner graph. GPCR improves GSR by rejecting the use of an external street map. The protocol consists of two parts: a restricted greedy forwarding procedure, which forwards the messages to the vehicles at a junction, and a repair strategy, which is based on the topology of real-world streets and junctions.

The anchor-based street and traffic-aware routing protocol (A-STAR) is designed exclusively for inter-vehicular communication systems operated in the city environment. A-STAR is dependent on data that is collected from city bus routes to determine an anchor path from the source node to the destination node with high connectivity for packet delivery [17]. The A-STAR routing protocol uses a more efficient recovery strategy for local maxima problems: It calculates new anchor paths for recovery and declares the void area causing the local maxima problem temporarily unreachable.

The connectivity-aware routing protocol (CAR) has distinctive features that enable it to maintain the cache of optimal route paths between numerous source and destination pairs. Predicting the position of the destination vehicle reformations route can be done by CAR in case there is a change in position [18].

The goal of the greedy traffic-aware routing protocol (GyTAR) is to achieve efficient use of network bandwidth and aims to achieve reduced end-to-end delay and packet loss. It adopts a new intersection-based geographical routing protocol capable of finding robust routes in a city environment with multi-lanes [19].

These protocols aim to transmit data packets to the destination as soon as possible. The basic expectations in the greedy approach of non-DTN routing protocols is that a vehicle transmits its packet to its neighbor, which is close to the destination. However, the forwarding strategy might fail if the neighbors are not closer to the destination than the vehicle. The individual recovery approach must be applied to overcome such failures.

\section{Delay-Tolerant Network (DTN)}

The delay-tolerant network (DTN) is a network approach that uses a carry-and-forward strategy related to a heterogeneous network to overcome the frequent disconnection of network nodes. When a vehicle cannot contact to other vehicles, DTN protocols store the packet information and then forward it later when an opportunity appears.

DTN uses a carry-and-forward strategy to overcome frequent disconnections of network nodes. In the carry-and-forward strategy, when a node cannot contact other nodes, it stores the packet, and the transfer is done based on some 
metrics from the neighboring nodes.

Vehicle-assisted data delivery (VADD) is based on the carry-and-forward approach using the predictable mobility of vehicles [20]. The main problem is to select a route for the forwarding path with minimal packet delivery delay using information about traffic patterns and road layout.

The motion vector routing algorithm (MOVE) was proposed for specialized sparse scenarios for V2I [21]. The MOVE algorithm uses knowledge of the velocities and the paths of neighboring vehicles to predict which vehicle is actually moving closest to an RSU destination. This algorithm assumes a network with lower density, in which infrequent opportunistic routing decisions must be made in a predictive manner. It is assumed that each vehicle knows its own direction and position, and the destination (RSU) is assumed to be a fixed location that is known globally. The current vehicular source node finds the closest distance between the current source and the destination along its path. The MOVE algorithm uses two main messages, HELLO and RESPONSE, for the carry-and-forward approach. The vehicle node regularly sends a HELLO beacon. The RESPONSE message is sent by neighboring nodes to enable the current vehicle to create the short route to the location along the path of the neighboring vehicle. Then, the current source vehicle decides to forward the message while establishing the current distance of each vehicle from the destination.

Geographic opportunistic routing (GeOpps) is considered a new type of delay-tolerant routing algorithm that uses the information available from the navigation system to route a data packet to a specific geographic location. This allows the selection of vehicles to carry the information closer to the final destination of the packets. The node with the minimum arrival time is selected to forward the packets. The GeOpps delivery rate depends on mobility patterns and road topology but not on vehicle density [22].

The geographical spray in the VDTN (GeoSpray) protocol uses the principles of single copy and single path GeOpps to carry out a multiple-copy, multiple-path routing approach [23].

Adaptive road-based routing (ARBR) assumes that each vehicle must know its geographical position and direction through GPS and is equipped with digital maps to select a road portion or an intersection [24]. The ARBR protocol uses two mechanisms to increase the delivery ratio and reduce end-to-end delay:

1) Find a high-quality route for forwarding between the route-requesting vehicle and the packet-forwarding vehicle along with the discovered route.

2) The stability of the routes is ensured by updating the route in the header of the road response packet through intermediate nodes.

MaxProp is used for sparse networks with limited transmission possibilities. It is based on prioritizing both the list of packets transmitted to others and the list of packets to be dropped. It operates in three basic stages: neighbor discovery stage, data transfer stage, and storage management stage [25].

Object pursuing based on efficient routing algorithm (OPERA) works in 
sparse situations, and it is applicable for both moving and fixed destinations [26]. The optimization of decision-making at intersections is based on the connectivity and feasibility metrics. By exploiting the related metrics, the next road is selected to forward the packet to minimize the overall delay. Position-based directional vehicular routing (PDVR) makes sure the packets can be sent to the destination in an efficient and stable route. It selects the next-hop from the vehicles traveling in the same direction as the forwarding vehicle based on their angular direction relative to the destination.

OPERA works in sparse cases and can be used for both moving vehicles and fixed destination (RSU). The optimization of decision-making at intersections is based on the feasibility and the connectivity metrics. By taking advantage of the associated metrics, to minimize the overall delay, the next street is selected to forward the packet. PDVR ensures that the packets can be forwarded to the destination in an efficient and stable route. Based on their angular direction relative to the destination, it selects the next-hop from the vehicles traveling in the same direction toward the destination as the forwarding vehicle.

\section{Simulation Results and Discussion}

Table 1 shows the parameters used in NS-2 simulation. These parameters were used in [2] to study the performance of non-DTN routing protocols in terms of percentage of delivery and End-to-End delay. In this paper the same parameters were used to compare the performance of DTN and non-DTN routing protocols.

Table 1. Simulation parameters.

\begin{tabular}{cc}
\hline \multicolumn{2}{c}{ Simulation parameters } \\
\hline Network simulator & NS-2 version 2.34 \\
Simulation Time & 300 seconds \\
Map Size & $2000 \mathrm{~m} \times 1500 \mathrm{~m}$ \\
Mobility Model & M-Grid \\
Vehicles speed & $20,40,60,80 \mathrm{~km}$ \\
Number of vehicles & $100-300$ \\
MAC protocol & $802.11 \mathrm{DCF}$ \\
Channel Capacity & $2 \mathrm{Mbps}$ \\
Trans. Range & $250 \mathrm{~m}$ \\
Traffic Model & 15 CBR connections \\
Packet sending rate & 4 packets/second \\
Data packet size & 128 bytes \\
Channel type & Wireless channel \\
Antenna Model & Omni directional \\
\hline
\end{tabular}


Figure 4 shows the node density versus the packet delivery ratio for non-DTN and DTN routing protocols. The packet delivery ratio for DTN is better than that for the non-DTN routing protocol in all node densities. the reason is that the DTN routing protocol delivers packets from the source to the destination whenever the algorithm finds a robust route in between, thus increasing the percentage of delivery ratio.

In addition, there is an overlap between GeOpps and GyTAR in node densities up to 250 vehicles $/ \mathrm{km}^{2}$, and then GeOpps outperforms the GyTAR routing protocol.

Figure 5 shows the node density versus the end-to-end delay. The performance for non-DTN routing protocols is superior to the performance of DTN routing protocols for all node densities. Delay routing protocols need to store packets before forwarding them to the destination. As a result of packets buffering and decision-making in the algorithms, extra processing and buffering time is needed compared to non-delay routing.

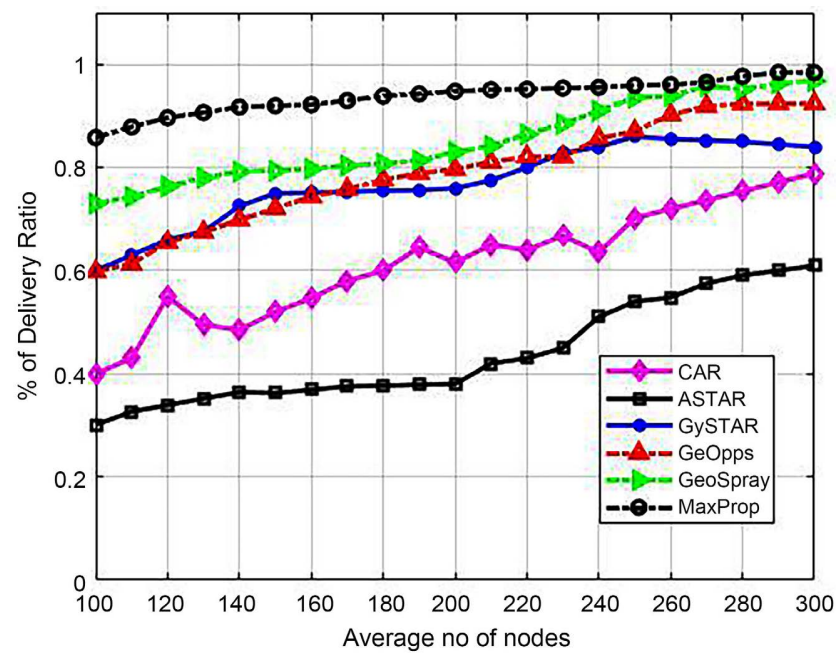

Figure 4. Node density vs. percent of delivery ratio.

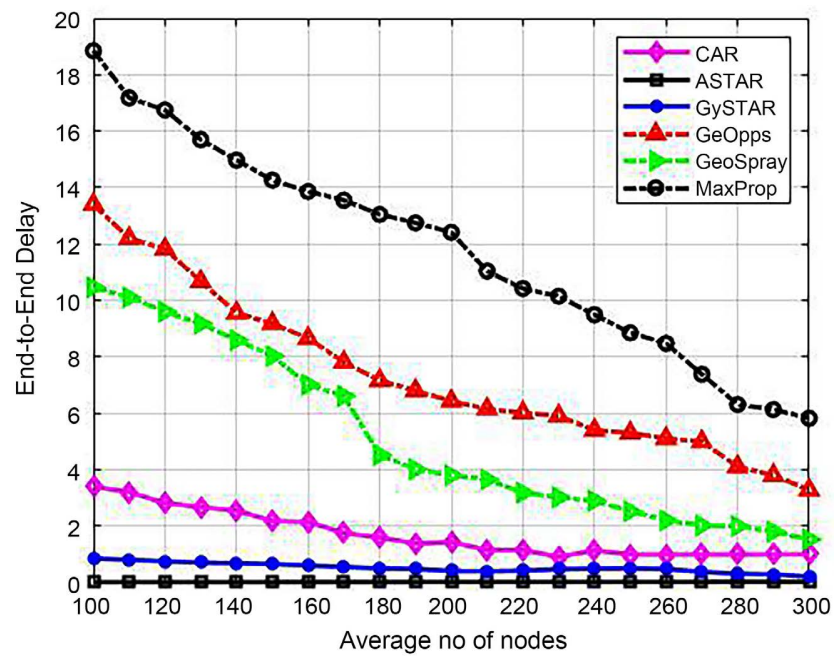

Figure 5. Node density vs. end-to-end delay. 


\section{Conclusions}

Performance measurements for non-DTN and DTN position-based routing protocols in VANETs depend on many factors, such as rapid topological changes, the mobility of vehicles, vehicular density, and other driving environments. Performance metrics also depend on the appropriate use of the mobility model and the propagation model. VANETs routing protocols are expected to perform well both in the city and on highways. In this paper, the two types of protocols belonging to unicast position-based routing protocols are discussed.

Simulation was conducted on the NS2 simulator. Simulations of non-DTN routing protocols (A-STAR, CAR, GyTAR) and DTN routing protocols (GeOpps, GeoSpray, MaxProp) were carried out. According to the simulation results, the DTN routing protocols outperform in delivery ratio compared to the non-DTN routing protocols, but they lead to more average delay due to buffering, the processing algorithm, and priority calculation.

In conclusion, non-DTN protocols are more suitable for the city environment since the distance between nodes is relatively smaller and the variations in the network topology are slower than they are on highways. On the other hand, DTN protocols are more suitable for highways due to the buffering of packets until a clear route to destination is available.

\section{Conflicts of Interest}

The authors declare no conflicts of interest regarding the publication of this paper.

\section{References}

[1] Venkatesh, I.A. and Murali, R. (2012) Vehicular Ad hoc Networks (VANETs): Issues and Applications. Journal of Analysis and Computation, 8, 31-46.

[2] Abdalla, A.M. (2018) Performance Evaluation for a Unicast Non Delay Tolerant Position Based Routing Protocols in VANETs. International Journal of Computer Applications, 180, 1-6. https://doi.org/10.5120/ijca2018916235

[3] Bilal, S.M., Bernardos, C.J. and Guerrero, C. (2013) Position-Based Routing in Vehicular Networks: A Survey. Journal of Network and Computer Applications, 36, 685-697. https://doi.org/10.1016/j.jnca.2012.12.023

[4] Sharef, B.T., Alsaqour, R.A. and Ismail, M. (2014) Vehicular Communication Ad Hoc Routing Protocols: A Survey. Journal of Network and Computer Applications, 40, 363-396. https://doi.org/10.1016/j.jnca.2013.09.008

[5] Venkatesh, Indra, A. and Murali, R. (2014) Routing Protocols for Vehicular Ad Hoc Networks (VANETs): A Review. Journal of Emerging Trends in Computing and Information Sciences, 5, 25-43.

[6] Cunha, F., Villas, L., Boukerche, A., Maia, G., Viana, A., Mini, R.A.F. and Loureiro, A.A.F. (2016) Data Communication in VANETs: Protocols, Applications and Challenges. Ad Hoc Networks, 44, 90-103. https://doi.org/10.1016/j.adhoc.2016.02.017

[7] Le, D.T., et al. (2021) A Behavior-Based Malware Spreading Model for Vehicle-to-Vehicle Communications in VANET Networks. Electronics, 10, 2403. https://doi.org/10.3390/electronics10192403 
[8] Al-Sultan, S., Al-Doori, M.M., Al-Bayatti, A.H. and Zedan, H. (2014) A Comprehensive Survey on Vehicular Ad Hoc Network. Journal of Network and Computer Applications, 37, 380-392. https://doi.org/10.1016/j.jnca.2013.02.036

[9] Lin, D., Kang, J., Squicciarini, A., Wu, Y.J., Gurung, S. and Tonguz, O (2017) MoZo: A Moving Zone Based Routing Protocol Using Pure V2V Communication in VANETs. IEEE Transactions on Mobile Computing, 16, 1357-1370. https://doi.org/10.1109/TMC.2016.2592915

[10] Mekki, T., Jabri, I., Rachedi, A. and ben Jemaa, M. (2017) Vehicular Cloud Networks: Challenges, Architectures, and Future Directions. Vehicular Communications, 9, 268-280. https://doi.org/10.1016/j.vehcom.2016.11.009

[11] Mahdi, H.F., Abood, M.S. and Hamdi, M.M. (2021) Performance Evaluation for Vehicular Ad-Hoc Networks-Based Routing Protocols. Bulletin of Electrical Engineering and Informatics, 10, 1080-1091. https://doi.org/10.11591/eei.v10i2.2943

[12] Jain, S., Fall, K. and Patra, R. (2004) Routing in a Delay Tolerant Network. ACM SIGCOM04, Portland, 30 August-3 September 2004, 145-158.

https://doi.org/10.1145/1015467.1015484

[13] Hossen, M.S. (2019) DTN Routing Protocols on Two Distinct Geographical Regions in an Opportunistic Network: An Analysis. Wireless Personal Communications, 108, 839-851. https://doi.org/10.1007/s11277-019-06431-W

[14] Paul, B., Md. Ibrahim and Md. Abu, N.B. (2011) VANET Routing Protocols: Pros and Cons. International Journal of Computer Applications, 20, 28-34.

https://doi.org/10.5120/2413-3224

[15] Kang, H., Ahmed, S.H., Kim, D. and Chung, Y.-S. (2015) Routing Protocols for Vehicular Delay Tolerant Networks: A Survey. International Journal of Distributed Sensor Networks, 11, 1-9. https://doi.org/10.1155/2015/325027

[16] Benamar, N., Singh, K.D., Benamar, M., El Ouadghiri, D. and Bonnin, J.-M. (2014) Routing Protocols in Vehicular Delay Tolerant Networks: A Comprehensive Survey. Computer Communications, 48, 141-158. https://doi.org/10.1016/j.comcom.2014.03.024

[17] Seet, B.-C., Liu, G.P., Lee, B., Foh, C., Juan Wong, K. and Lee, K.-K. (2004) A-STAR: A Mobile Ad Hoc Routing Strategy for Metropolis Vehicular Communications. Proceedings IFIP TC6 Networking, Vol. 3042, 989-999. https://doi.org/10.1007/978-3-540-24693-0 81

[18] Naumov, V. and Gross, T.R. (2007) Connectivity-Aware Routing (CAR) in Vehicular Ad-Hoc Networks. IEEE INFOCOM 2007 26th IEEE International Conference on Computer Communications, Anchorage, 6-12 May 2007, 1919-1927. https://doi.org/10.1109/INFCOM.2007.223

[19] Jerbi, M., Senouci, S.-M., Meraihi, R. and Ghamri-Doudane, Y. (2007) An Improved Vehicular Ad Hoc Routing Protocol for City Environments. IEEE International Conference on Communications, Glasgow, 24-28 June 2007, 3972-3979. https://doi.org/10.1109/ICC.2007.654

[20] Zhao, J. and Cao, G.H. (2008) VADD: Vehicle-Assisted Data Delivery in Vehicular Ad Hoc Networks. IEEE Transactions on Vehicular Technology, 57, 1910-1922. https://doi.org/10.1109/TVT.2007.901869

[21] Zhao, Y. (2005) Motion Vector Routing Protocol: A Position Based Routing Protocol for Mobile Ad Hoc Networks. The University of Arizona, Tucson.

[22] Leontiadis, I. and Mascolo, C. (2007) GeOpps: Geographical Opportunistic Routing for Vehicular Networks. Proceedings of the IEEE International Symposium on a World of Wireless, Mobile and Multimedia Networks (WOWMOM07), Espoo, 
18-21 June 2007, 1-6. https://doi.org/10.1109/WOWMOM.2007.4351688

[23] Soares, V.N.G.J., Rodrigues, J.J.P.C. and Farahmand, F. (2014) GeoSpray: A Geographic Routing Protocol for Vehicular Delay-Tolerant Networks. Information Fusion, 15, 102-113. https://doi.org/10.1016/j.inffus.2011.11.003

[24] Arzil, S.A., Aghdam, M.H. and Ali Jabraeil Jamali, M. (2010) Adaptive Routing Protocol for VANETs in City Environments Using Real-Time Traffic Information. 2010 International Conference on Information, Networking and Automation (ICINA), Vol. 2, V2-132-V2-136. https://doi.org/10.1109/ICINA.2010.5636966

[25] Burgess, J., et al. (2006) MaxProp: Routing for Vehicle-Based Disruption-Tolerant Networks. INFOCOM: International Conference on Computer Communications, Vol. 6, 1-11. https://doi.org/10.1109/INFOCOM.2006.228

[26] Abuelela, M., Olariu, S. and Stojmenovic, I. (2008) OPERA: Opportunistic Packet Relaying IN Disconnected Vehicular as Hoc Networks. 5th IEEE International Conference on Mobile Ad Hoc and Sensors Systems, Atlanta, 29 September-2 October 2008, 285-294. https://doi.org/10.1109/MAHSS.2008.4660077 